\title{
Análisis bibliométrico de la Revista Colombiana de Ciencia Animal - RECIA 2009-2018. Indicadores de producción
}

\author{
Bibliometric analysis of the Colombian Journal of Animal Science - \\ RECIA 2009-2018. Production indicators
}

De La Ossa J, Montes-Vergara D, González TM, Salgado AL. Análisis bibliométrico de la Revista Colombiana de Ciencia Animal - RECIA 2009-2018. Indicadores de producción. Rev Colombiana Cienc Anim. Recia. 2019; 11(1):Articulo724. DOI: https://doi.org/10.24188/recia.v11.n1.2019.724

Universidad de Sucre, Colombia

Los autores permiten a RECIA reimprimir el material publicado en él. En caso de que un autor quiera traducir o usar una publicación parcial o completa de nuestro Diario, el autor debe obtener un permiso por escrito del editor de la revista.

Revista Colombiana de Ciencia Animal - RECIA está distribuido bajo una Licencia Creative Commons Atribución-CompartirIgual 4.0 Internacional. 


\title{
Análisis bibliométrico de la Revista Colombiana de Ciencia Animal - RECIA 2009-2018. Indicadores de producción
}

\author{
Bibliometric analysis of the Colombian Journal of Animal Science - RECIA 2009-2018. \\ Production indicators
}

Jaime De La Ossa V. Ph.D.

DOI: https://doi.org/10.24188/recia.v11.n1.2019.724

Editor Revista Colombiana de Ciencia Animal, Universidad

de Sucre, Facultad de Ciencias Agropecuarias, Colombia.

(i) https://orcid.org/0000-0002-9374-7765

jaime.delaossa@unisucre.edu.co

Donicer Montes-Vergara. Ph.D.

Editor Asociado, Universidad de Sucre, Facultad de Ciencias

Agropecuarias, Colombia

(i) https://orcid.org/0000-0002-2860-0505

donicer.montes@unisucre.edu.co

Marco González Tous. M.Sc.

Universidad de Córdoba, Montería, Colombia.

(i) https://orcid.org/0000-0001-6500-5601

marcogonzaleztous@gmail.com

Luis Salgado-Arroyo. Ing.

Editor Técnico, Universidad de Sucre, Sincelejo, Colombia

(D) https://orcid.org/0000-0003-0738-9226

reciaeditor@unisucre.edu.co

\section{RESUMEN}

Objetivo. Realizar un análisis bibliométrico de la producción científica publicada en la Revista Colombiana de Ciencia Animal 2009-2018. Materiales y métodos. Se realizó un estudio bibliométrico descriptivo-retrospectivo incluyendo los artículos publicados en los últimos 10 años de la revista. Se seleccionaron indicadores bibliométricos como distribución cronológica, producción científica, índice de crecimiento, categorización temática, productividad de autores, coautoría y cooperación institucional. Se calcularon los indicadores cienciométricos mediante el software Harzing's Publish or Perish 6, tomando como base de datos el Google Académico. Resultados. Fueron evaluados 379 artículos científicos y se publicó un promedio de 16.48 artículos/volumen. La productividad ponderada fue de 0.39 artículos/autor; el H-index fue 13; el g-index fue de 18; el hi normalizado fue de 8 y el h1 anual fue de 0.89 . Se evidenció un incremento en las contribuciones internacionales con predominio de los artículos originales. Conclusiones. Publicar un mayor número de artículos en inglés constituiría una oportunidad de mejorar la visibilidad de la revista. Se mostraron los indicadores específicos de la revista teniendo en cuenta su visibilidad y productividad ofrecidos por el programa Publish or Perish.

Palabras clave: Análisis bibliométrico, Harzing's Publish or Perish, producción científica, RECIA. 


\begin{abstract}
Objective. Perform a bibliometric analysis of the scientific production published in the Colombian Journal of Animal Science 2009-2018. Materials and methods. A descriptive-retrospective bibliometric study was carried out including the articles published in the last 10 years of the journal. Bibliometric indicators were selected as chronological distribution, scientific production, growth index, thematic categorization, author productivity, co-authorship and institutional cooperation. The scientometric indicators were calculated using the Harzing's Publish or Perish 6 software, based on the Google Scholar. Results. 379 scientific articles were evaluated and an average of 16.48 articles / volume was published. The weighted productivity was 0.39 articles / author; the $\mathrm{H}$-index was 13; the g-index was 18; the normalized hi was 8 and the annual h1 was 0.89 . There was an increase in international contributions with predominance of original articles. Conclusions. Publishing a greater number of articles in English would be an opportunity to improve the visibility of the journal. The specific indicators of the journal were shown taking into account their visibility and productivity offered by the Publish or Perish program.
\end{abstract}

Keywords: Bibliometric analysis, Harzing’s Publish or Perish, scientific production, RECIA.

\title{
INTRODUCCIÓN
}

La bibliometría es la ciencia interdisciplinaria que permite el análisis cuantitativo de la producción científica y facilita reconocer el valor real que tiene la actividad científica en determinada disciplina por métodos matemáticos y estadísticos (1). En siglo XX (1917) fueron reportadas las primeras bibliometrías por Cole y Eales (2), quines estudiaron el crecimiento de la literatura en anatomía comparada a través de bibliografía. En 1969, el famoso científico británico, Allen Richard, propuso por primera vez el término "bibliometría" en lugar de "bibliografía estadística". La aparición de este término marcó el nacimiento formal de la bibliometría (3).

Actualmente se viene presentando un alto crecimiento en su uso debido a que permite a investigadores el cálculo y en el análisis de los valores de lo que es cuantificable en la producción y en el consumo de la información científica, estudiando áreas específicas mediante el análisis de citas, publicaciones, distribución geográfica, coautoría, etc. $(3,4)$. También se utiliza para evaluar el desempeño investigativo de instituciones, investigadores y revistas, así como los campos de investigación.

El uso de indicadores bibliométricos se basa en que las publicaciones científicas son un resultado esencial de la actividad académica e investigativa de una institución o un país. Un nuevo conocimiento adquiere valor cuando se da a conocer y se difunde, ya que es así como progresa la ciencia (4). Es por ello que permite caracterizar, tanto en términos cuantitativos como cualitativos, la producción científica de un individuo, institución o país mediante el empleo de parámetros como: cantidad, factor de impacto (FI) y citas de los artículos publicados $(5,6)$.

Los estudios bibliométricos aplicados a revistas científicas han sido y serán objeto de grandes debates por la marcada importancia que tiene analizar la producción científica de las mismas.

\section{Historia de la Revista Colombiana de Ciencia Animal - RECIA.}

RECIA nace como una iniciativa de los docentes de la Facultad de Ciencias Agropecuarias de la Universidad de Sucre, Colombia, quienes vieron en la divulgación de trabajos científicos una gran oportunidad de integración con los investigadores del área y una forma expedita de dar a conocer los resultados y labores investigativas que se desarrollaban. Esta iniciativa se logró concretar en el mes de enero de año 2009 con la publicación del primer número.

En el editorial, que inició la "vida pública" de RECIA, se señaló que se le entrega a la sociedad una revista de divulgación científica planeada, discutida y creada en la Facultad de Ciencias Agropecuarias de la Universidad de Sucre, especialmente si se tenía en cuenta que habían transcurrido 54 años desde 
1955 hasta la fecha, para que un medio escrito de divulgación de la productividad intelectual científica se volviera a publicar por estas tierras; se recordaba con esta frase, que antes y como trabajo de profesionales locales y otros extranjeros, había existido en este lar, la Revista de la Sociedad Lineana.

Al respecto de la Sociedad Lineana, es valioso destacar que fue conformada, por: George Dahl, José Nicolás Anaya, Luis Arévalo Salazar, Dimas Badel, Carlos Bitar, William Bustillo Fernández, José de J. Corrales, Dennis R. Crespo, Enrique Díaz, Carlos Angulo Escalante, Eduardo Espinosa U., Clemencia Esquivel Delgado, Jorge Fadul, Luis Eduardo Giraldo López, Rodrigo Giraldo López, José I. Granados de la Hoz, Julio Alejandro Hernández S., Rafael Hernández M., Alina Olivares Prado, Ernesto Panezzo Robledo, Ángel Quintana, Olga Quintero Caraballo, Arcesio Rueda Llorente, Carlos Serrano Navas, Francisco Tamara Sierra, Edmundo Yunis y Eduardo Yunis, y sus socios honorarios Paul Rivet, Angel Dotor, Philips Hershkovitz y Luis López de Mesa.

La Revista Colombiana de Ciencia Animal (Colombian Journal of Animal Science) - RECIA, es una publicación científica patrocinada por la Facultad de Ciencias Agropecuarias de la Universidad de Sucre, Colombia, sin ánimo de lucro, se edita ininterrumpidamente desde el año 2009 con una periodicidad fija semestral, abierta a la comunidad internacional y dirigida a los investigadores que buscan divulgar información científica de calidad académica y que están dispuestos a someter sus obras ante pares evaluadores externos.

Publica artículos originales, notas cortas, estudios de casos y revisiones bibliográficas, preparados por investigadores del orden nacional e internacional, que contribuyen con sus trabajos en las áreas de la ciencia animal, medicina veterinaria, zootecnia, biología productiva, extensión rural, manejo e investigación en fauna silvestre, conservación, ecología, agroecología, ciencias agrícolas y microbiología del suelo y otros tópicos que a juicio del comité editorial sean de interés general.

Por todo lo anteriormente planteado, el objetivo de este estudio fue evaluar producción científica publicada en la Revista Colombiana de Ciencia Animal, durante el período 2009-2018.

\section{MATERIALES Y MÉTODOS}

Tipo de estudio. Se realizó un estudio bibliométrico descriptivo-retrospectivo sobre la producción científica de la Revista Colombiana de Ciencia Animal - RECIA durante el período 2009-2018.

Variables. Se estudiaron las siguientes variables: total de números, total de artículos, todas de autores, nacionalidad de autores, citas, promedio de citas por año, promedio de citas por artículos, promedio de artículos por autor, promedio de autores por artículo, índice h, índice g, índice h contemporáneo, índice h individual (hI), índice hI normalizado, índice AWCR, índice AW, índice AWCRpA, índice e, índice hm, índice hI anual, amplitud H, amplitud G, tipología documental, filiación de los autores.

Índice $\mathbf{H}$. Considera tanto la cantidad de artículos como la cantidad de citas que reciben. Un índice h de 20 significa que un académico ha publicado al menos 20 artículos que han recibido al menos 20 citas cada uno. El índice h combina una evaluación de la cantidad (número de artículos) y una aproximación de la calidad (impacto o citas de estos documentos).

Índice g. El índice g se calcula en función de la distribución de las citas recibidas por las publicaciones de un investigador determinado. Un índice g de 20 significa que el académico ha publicado al menos 20 artículos que combinados han recibido al menos 400 citas. Sin embargo, a diferencia del índice h, estas citas podrían generarse solo por un pequeño número de artículos. Por ejemplo, un académico con 20 artículos, 15 de los cuales no tienen citas y los cinco restantes tienen respectivamente 350, $35,10,3$ y 2 citas tendrían un índice g de 20, pero un índice h de 3 (tres artículos con al menos 3 citas cada una).

Índice contemporáneo hc: Toma en cuenta el tiempo de vida de los artículos, y a medida que pasa el tiempo, este índice decrece. 
Índice $\mathbf{h}$ individual o hI. Referido al número de autores promedio con que publica el autor, con el fin de particularizar el impacto de los miembros de un equipo de colaboradores. Se obtiene dividiendo $\mathrm{h}$ por el número medio de autores en los h trabajos.

Índice individual normalizado, hI_norm. Referido a las citaciones hechas a cada artículo. Es normalizar el número de citas para cada artículo dividiendo el número de citas por el número de autores de ese documento y luego calcular el índice h de los recuentos de referencias normalizadas.

Índice hm. Toma en cuenta el número total de citaciones al autor.

Índice e. Para diferenciar aquellos autores que tienen trabajos que reciben muchas citas y que, sin embargo, pudieran mostrar un índice h igual al resto.

Índice $\mathbf{h}$ anual, hIanual. Para analizar el impacto anual de la revista. Mide el número medio de un solo autor (o revista) señala índice h equivalentes que un académico (o revista) ha acumulado en cada año.

Cada uno de estos indicadores está explicado con precisión en el tutorial del programa Publish or Perish (7).

Documentos. Se consideraron todos los artículos originales, revisiones de literatura, casos clínicos, notas cortas y editoriales; se excluyeron los artículos de opinión. Se consideró como país de origen el citado por el autor.

Recolección de datos y procedimiento. Se realizó la búsqueda de todos los artículos publicados en la web de la revista. Además, se realizó la consulta a través de indicadores cienciométricos que ofrece el programa Publish or Perish 6.0., digitando en la caja de navegación del Publish or Perish el nombre de la revista, su ISSN (International Standard Serial Number):2027-4297. También se debe establecer el periodo de estudio; el cual puede ser de tres tipos: 1) análisis del impacto del autor (author impact analysis), 2) análisis del impacto de la revista (journal impact analysis) y 3) búsqueda de citas generales (general citation search). También permite copiar y exportar los resultados estadísticos y las referencias bibliográficas en BibTex y otros formatos (7).

\section{RESULTADOS}

Se analizaron 10 volúmenes del 1(1): 2009 al 10(2): 2018, con 22 números en total, relacionado 379 artículos, de los cuales 22 (6\%) editoriales, 223 (59\%) artículos originales, 43 (11\%) revisiones de literatura, 35 (9\%) casos clínicos y 55 (15\%) notas cortas (Figura 1).

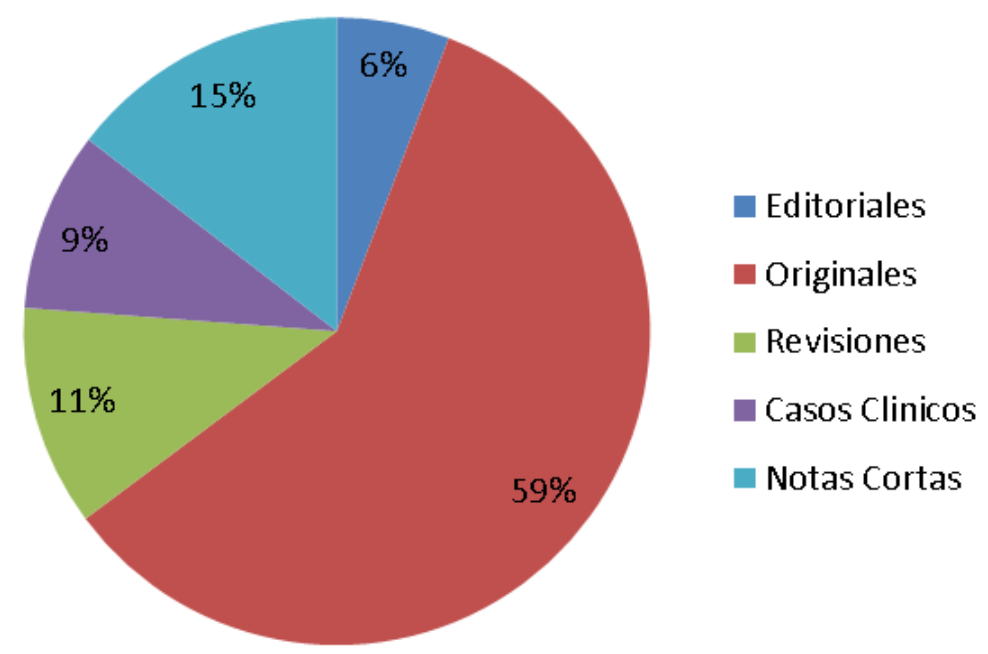

Figura 1. Tipología de los artículos publicados en la revista periodo 2009-2018. 
El número de artículos publicados por año fue variable, en promedio se publicaron 16.48 artículos por año. Con relación a los autores y su origen, se encontró que 417 de los autores con filiación interna o local, es decir, que provienen de la misma institución donde se edita la revista. También se encontró que 406 de los autores son de origen nacional (Colombia) y 372 autores registran filiación extranjera o internacional. Como se puede observar en la figura 2, se destaca que los autores nacionales e internacionales fueron aumentando e incluso llegaron a desplazar la contribución de origen local.

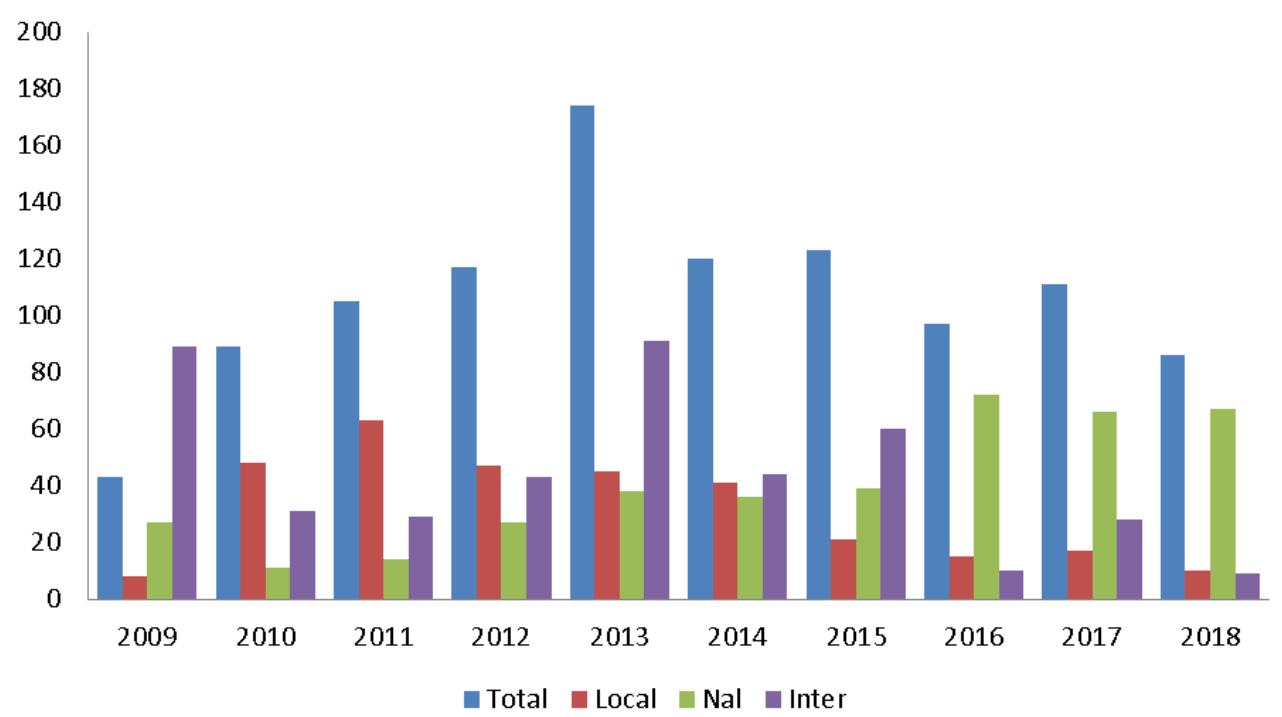

Figura 2. Autores de acuerdo con el origen de la filiación.

El índice de colaboración (cociente entre el número de firmas y el número de trabajos) por año osciló entre un mínimo de 1,59 y un máximo de 3,48 autores por artículos, para los años 2009 y 2013 respectivamente.

\section{Índice de colaboración}

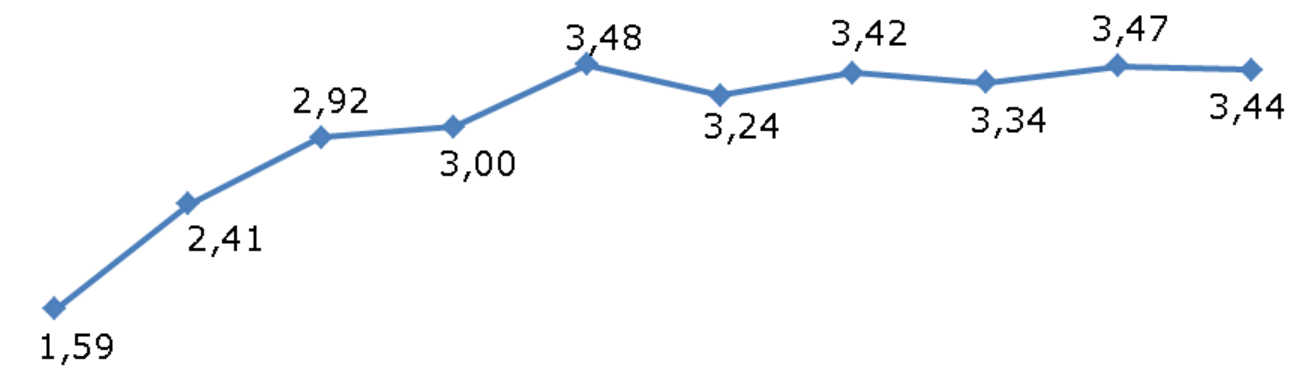

$\begin{array}{llllllllll}2009 & 2010 & 2011 & 2012 & 2013 & 2014 & 2015 & 2016 & 2017 & 2018\end{array}$

Figura 3. Índice de colaboración del periodo 2009-2018.

Luego se realizó una consulta con el software Publish or Perish 6.0 (7), con periodo de observación (2009-2018), arrojando una consulta total de 536 elementos, 893 citaciones, índice h de 13 e índice g de 18 como se observa en la tabla 2. 
Tabla 2. Indicadores de la Revista Colombiana de Ciencia Animal - Recia, Periodo 2009-2018.

\begin{tabular}{lc}
\hline \multicolumn{1}{c}{ Indicadores } & Cifras \\
\hline Reference date: & $31 / 03 / 201909: 35: 28$ a.m. \\
Publication years: & $2009-2019$ \\
Citation years: & $10(2009-2019)$ \\
Papers: & 619 \\
Citations: & 1059 \\
Citations/year: & 105.90 \\
Citations/paper: & 1.71 \\
Citations/author: & 428.24 \\
Papers/author: & 265.32 \\
Authors/paper: & $2.76 / 3.0 / 3($ mean $/$ median $/$ mode $)$ \\
Age-weighed citation rate: & 162.21 (sqrt=12.74), 64.32/author \\
Hirsch h-index: & $19(\mathrm{a}=6.27, \mathrm{~m}=1.30,297$ cites $=28.0 \%$ coverage $)$ \\
Egghe g-index: & $1.46,373$ cites=35.2\% coverage $)$ \\
PoP hI,norm: & 7 \\
PoP hI,annual: & 0.70 \\
\hline
\end{tabular}

Fuente: Harzing (7).

Igualmente se realizó una verificación de los artículos más citados en este periodo, con el mismo programa Publish or Perish (7), De esta manera, se encontró que el artículo más citado con 34 citas fue "Hongos formadores de micorrizas arbusculares: una alternativa biológica para la sostenibilidad de los agroecosistemas de praderas en el Caribe colombiano", publicado en el Volumen 3 número 2 del año 2011 (Tabla 3).

Luego de la actualización de la plataforma OJS a su última versión a comienzos del 2018 se implementó herramientas que permiten ver el crecimiento dinámico de las visitas al sitio web de la revista (Figura 4) y además ha posibilitado el acceso a la producción científica de los profesionales desde cualquier lugar y momento. Es por ello que constituye una necesidad cada vez más creciente, el evaluar los procesos de producción y comunicación del conocimiento científico resultante de la actividad investigativa.

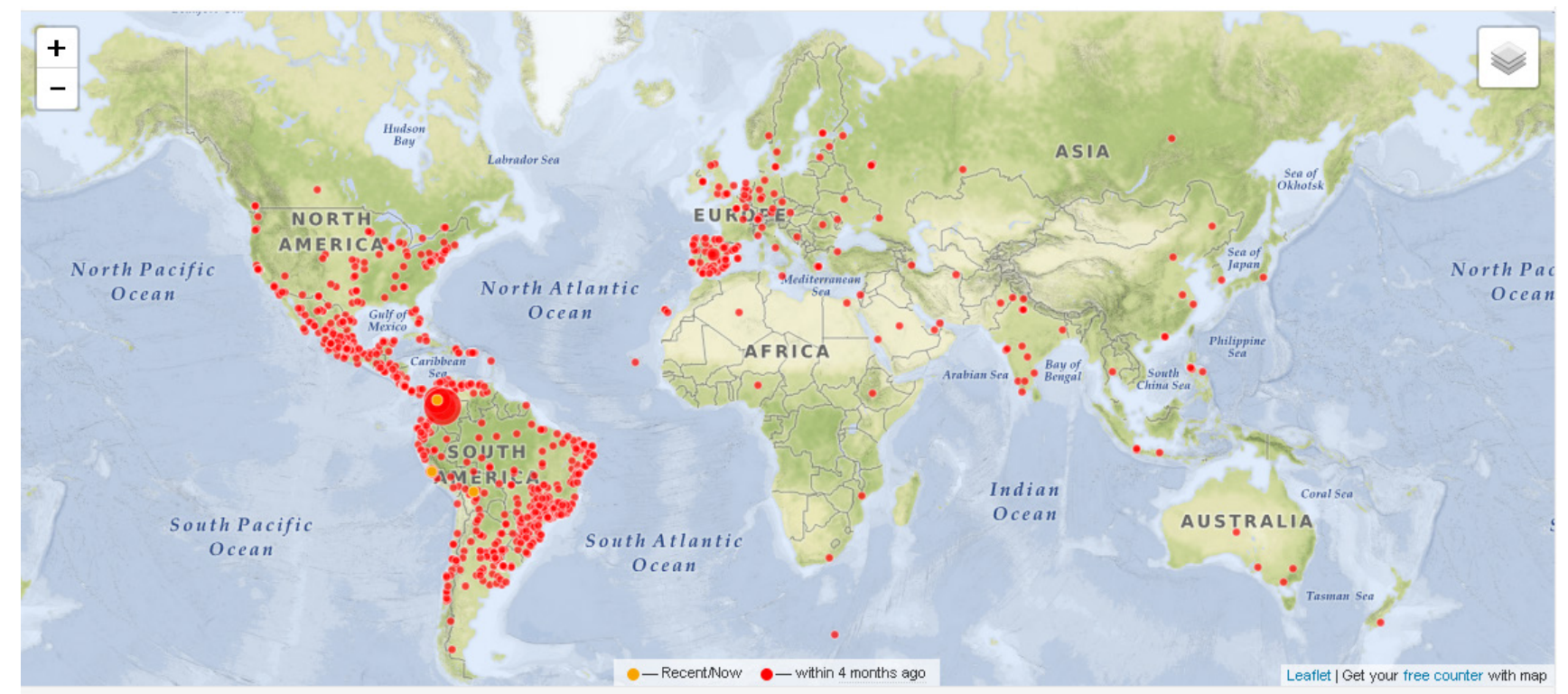

Figura 4. Geolocalización de las visitas de la revista desde su implementación con la actualización del OJS (Junio-Octubre; 2018). 
Tabla 3. Artículos más citados en el periodo 2009-2017.

\begin{tabular}{|c|c|c|c|}
\hline Citas & Autores & Titulo & Años \\
\hline 34 & A Pérez, JR Sierra, Montes V & $\begin{array}{l}\text { Hongos formadores de micorrizas arbusculares: una alternativa biológica para la } \\
\text { sostenibilidad de los agroecosistemas de praderas en el caribe colombiano }\end{array}$ & 2011 \\
\hline 29 & A Arrieta, VD Montes & $\begin{array}{l}\text { Alfabetización digital: uso de las TIC’s más allá de una formación instrumental y una } \\
\text { buena infraestructura }\end{array}$ & 2011 \\
\hline 28 & S Galván-Guevara & Biodiversidad en el área de influencia de la estación primates de Colosó, sucre, Colombia. & 2009 \\
\hline 26 & AC Cuéllar, RH Yunus & $\begin{array}{l}\text { Evaluation of the yield and the antimicrobial activity ofthe essential oils from: Eucalyptus } \\
\text { globulus, Cymbopogon citratus and Rosmarinus officinalis in mbarara district ... }\end{array}$ & 2009 \\
\hline 23 & $\begin{array}{l}\text { J Figueroa, J Salcedo, Y } \\
\text { Aguas... }\end{array}$ & $\begin{array}{l}\text { Recubrimientos comestibles en la conservación del mango y aguacate, y perspectiva, al } \\
\text { uso del propóleo en su formulación }\end{array}$ & 2011 \\
\hline 20 & K Cury, A Martínez, Aguas & Caracterización de carne de conejo y producción de salchicha & 2011 \\
\hline 18 & S galván-Guevara & $\begin{array}{l}\text { Herpetofauna registrada para el área de influencia de la reserva forestal protectora } \\
\text { serranía de Coraza, Colosó, Sucre, Colombia }\end{array}$ & 2009 \\
\hline 17 & E cervantes, A Espitia & Viabilidad de los sistemas bufalinos en Colombia & 2010 \\
\hline 16 & AC Cuéllar, J González & $\begin{array}{l}\text { Obtención del glucósido flavonoide Gossypitrina de los pétalos de las flores de Talipariti } \\
\text { elatum SW y evaluación de su posible efecto antioxidante }\end{array}$ & 2010 \\
\hline 15 & $\begin{array}{l}\text { VJ de la Ossa, A Pérez, PR } \\
\text { Patiño... }\end{array}$ & La investigación formativa como una necesidad en el pregrado & 2012 \\
\hline 15 & Jaime De La Ossa & Cacería de subsistencia en san marcos, sucre, colombia & 2011 \\
\hline 15 & $\begin{array}{l}\text { L Botero, J de la Ossa, PA } \\
\text { Espitia... }\end{array}$ & Importancia de los jagüeyes en las sabanas del Caribe colombiano & 2009 \\
\hline 14 & $\begin{array}{l}\text { PJ Díaz, PCE Molano, JC } \\
\text { Gaviria }\end{array}$ & $\begin{array}{l}\text { Diversidad genérica de hormigas (Hymenoptera: Formicidae) en ambientes de bosque } \\
\text { seco de los Montes de María, Sucre, Colombia }\end{array}$ & 2009 \\
\hline 13 & C Cuellar, OD Okori & $\begin{array}{l}\text { Preliminary phytochemical and antimicrobial evaluation of the fresh and dried whole } \\
\text { plant extracts from Commelina benghalensis. }\end{array}$ & 2010 \\
\hline 13 & M Niño-Puello & El inglés y su importancia en la investigación científica: algunas reflexiones & 2013 \\
\hline 11 & $\begin{array}{l}\text { JP Lima, CBP Juárez, AS } \\
\text { Teixeira... }\end{array}$ & $\begin{array}{l}\text { Seleção de locais de desova e sobrevivência de ninhos de quelônios Podocnemis no } \\
\text { baixo Rio Purus, Amazonas, Brasil }\end{array}$ & 2009 \\
\hline 11 & L Botero, J De la Ossa & $\begin{array}{l}\text { Estudio de caso: un sistema de producción con enfoque agroecológico, Departamento } \\
\text { del Magdalena, Colombia }\end{array}$ & 2010 \\
\hline 11 & $\begin{array}{l}\text { IL Navarro, AK Roman, FH } \\
\text { Gómez... }\end{array}$ & $\begin{array}{l}\text { Primer registro de Digitonthophagus gazella (Fabricius, 1787) para el departamento de } \\
\text { Sucre, Colombia }\end{array}$ & 2009 \\
\hline 11 & I Luis Navarro... & $\begin{array}{l}\text { Listado de escarabajos coprófagos (Coleoptera: Scarabaeidae: Scarabaeinae) de la } \\
\text { serranía de Coraza, Sucre (Colombia) }\end{array}$ & 2011 \\
\hline 11 & C Pérez, E Saavedra & $\begin{array}{l}\text { Avances en el manejo integrado de la bacteria Burkholderia glumae en el cultivo de } \\
\text { arroz en el Caribe colombiano }\end{array}$ & 2011 \\
\hline
\end{tabular}

\section{DISCUSIÓN}

La Revista Colombiana de Ciencia Animal - RECIA es una revista científica que se publica semestralmente, es de libre acceso, sin ánimo de lucro, fundada en 2009, arbitrada por expertos nacionales y extranjeros. Su área principal es la veterinaria pero además cubre los siguientes tópicos: ciencia animal, medicina veterinaria, zootecnia, biología productiva, extensión rural, manejo e investigación en fauna silvestre, conservación, ecología, agroecología, ciencias agrícolas 
y microbiología del suelo y otros tópicos que a juicio del comité editorial sean de interés general. Abierta a la comunidad internacional y dirigida a los investigadores que buscan divulgar información científica de calidad académica.

Los idiomas más utilizados son el español y el portugués y son una constante en las revistas de ciencias veterinarias latinoamericanas, donde la mayoría de los autores son del área o de la región. Este elemento, junto con los cambios observados en los tipos de documentos publicados, debe ser tenido en cuenta debido a que los indicadores de impacto van relacionados con el idioma de publicación ya que los artículos de investigación original y de revisión en inglés son los más citados (8-13).

Se puede concluir que la Revista RECIA es una publicación internacional que presenta indicadores bibliométricos similares a los de las revistas latinoamericanas representativas del área, con mejoras en los últimos años que la llevan a cumplir totalmente con los criterios editoriales internacionales. Esas mejoras, como el incremento en la proporción de artículos de investigación original y de revisión, son sin dudas fruto de la adecuada gestión editorial, que incluye, además, un bajo porcentaje de autocitas y de publicaciones de la misma institución.

De lo anterior se deduce que el número de citas recibidas, así como la importancia o el prestigio de la revista donde dichas citas son generadas, van en ascenso. Ello pudiera atribuirse a que la revista se encuentra indexada en diferentes bases de datos de reconocido prestigio internacional que aumentan su visibilidad, díganse: SciELO, CAB Abstrac, CAPES, EBSCO, Ulrich's, Crossref, Latindex, DOAJ, AMELI.CA, REDIB, ROAD, Dialnet, Index Copernicus, Actualidad Iberoamericana, OCLC, BASE, HINARI, AGORA, MIAR, Mendeley, OAJI entre otros índices, repositorios y bibliotecas nacionales e internacionales. Además la revista se encuentra en evaluación en Scopus e WoS para su inclusión en estas prestigiosas bases de datos.

Por otra parte la Revista RECIA es de acceso abierto, otorgando un acceso inmediato y sin restricciones ni embargos de ningún tipo en todo sus contenidos.

En conclusión el trabajo representa una evaluación bibliométrica de la revista RECIA, utilizando una metodología avalada por trabajos similares, necesaria para poder conocer indicadores de impacto que contribuyan a mejorar su calidad mediante la generación de una política editorial de vanguardia. Estos indicadores deben constituir un punto de partida para continuar el trabajo editorial que se viene desarrollando y buscar que la Revista Colombiana de Ciencia Animal - RECIA, se constituya en una revista de consulta obligada por investigadores, gobernantes y cibernautas en general.

\section{REFERENCIAS}

1. Merigó JM, Cancino C, Coronado F, Urbano D. Academic research in innovation: A country analysis. Scientometrics. 2016; 108(2):559-593. DOI: https://doi.org/10.1007/s11192-016-1984-4

2. Cole FJ, Eales NB. The history of comparative anatomy. Sci Prog 1917; 11(44):578-596. https:// www.jstor.org/stable/43426882

3. Liao H, Tang M, Luo L, Li C, Chiclana F, Zeng XJ. A Bibliometric Analysis and Visualization of Medical Big Data Research. Sostenibilidad. 2018; 10(1):166. DOI: http://dx.doi.org/10.3390/ $\underline{\text { su10010166 }}$

4.Patrón Carina,LópezJordi M.delCarmen,Piovesan Sylvia, Demaría Bettina.Análisis bibliométrico dela producción científica de la revista Odontoestomatología. Odontoestomatología. 2014; 16(23):3443. http://www.scielo.edu.uy/scielo.php?script=sci arttext\&pid=S1688-93392014000100005

5. Borrego Á. Altmétricas para la evaluación de la investigación y el análisis de necesidades de información. El profesional de la información. 2014; 23:352-357. DOI: https://doi.org/10.3145/ epi.2014.jul.02 
6. Gallardo Sánchez Y, Gallardo Arzuaga RL, Fonseca Arias M, Pérez Atencio ME. Caracterización cienciométrica de la producción científica de MEDWAVE, 2010-2014. Medwave. 2016; 16(8):e6538. DOI: https://doi.org/10.5867/medwave.2016.08.6538

7. Harzing AW. Publish or Perish [Internet]. London: Middlesex University; 2007. [consultado 18 abril 2019]. Disponible en: http://www.harzing.com/pop.htm

8. Mattar VS, González TM, Salgado AL. Análisis de las universidades colombianas de acuerdo con el ranking SCImago 2010-2012. Rev MVZ Córdoba. 2013; 18(1):3399-3407. https://doi. org/10.21897/rmvz.203

9. Ruano-Ravina A, Álvarez-Dardet C. Evidencebased editing: factors influencing the number of citations in a national journal. Ann Epidemiol. 2012; 22(9):649-653. https://doi.org/10.1016/j. annepidem.2012.06.104

10. Cremades Pallas R, Burbano P, Valcárcel de la Iglesia MA, Burillo-Putze G, Martín-Sánchez FJ, Miró O. Impacto de la inclusión de artículos escritos en inglés en revistas biomédicas españolas de edición multilingüe. An Sist Sanit Navar. 2013; 36(3):467-470. http://dx.doi.org/10.4321/ $\underline{\mathrm{S} 1137-66272013000300011}$

11. Sanz-Valero J, Casterá Vicente T, Wanden-Berghe C. Estudio bibliométrico de la producción científica publicada por la Revista Panamericana de Salud Pública/ Pan American Journal of Public Health en el período de 1997 a 2012. Rev Panam Salud Pública. 2014; 35(2):81-88. http://iris.paho.org/xmlui/bitstream/handle/123456789/8465/a01v35n2.pdf?sequence=1

12. Pérez-Anaya O, Ceballos-Ospino G, González-Gélvez D, Suescún-Arregocés J. Análisis bibliométrico de la revista Duazary en el quinquenio 2012-2016. Duazary. 2017; 14 (2):122-130. Doi: http:// dx.doi.org/10.21676/2389783X.197

13. Carreño LM, Poutou PRA, Mattar S, González T. Indicadores bibliométricos de actividad de la revista MVZ Córdoba 1994-2008. Rev MVZ Córdoba. 2009; 14(1): 1531-1543. https://doi. org/10.21897/rmvz.363 